\title{
Mitbetreuung und Einbeziehung von Angehörigen in Comprehensive Cancer Centern - eine Erhebung der AG Palliativmedizin der von der Deutschen Krebshilfe geförderten Onkologischen Spitzenzentren
}

\section{Support for and involvement of family caregivers in Comprehensive Cancer Center - an Assessment of the Palliative Care Working Group within the network of Comprehensive Cancer Center funded by the German Cancer Aid}

\section{(ㄷ) (ㄱ) $(9$}

Autoren

Karin Oechsle ${ }^{1}$, Tabea Theißen ${ }^{1}$, Maria Heckel ${ }^{2}$, Lisa Schwenzitzki ${ }^{1}$, Anneke Ullrich ${ }^{1}$, Christoph Ostgathe ${ }^{2}$

Institute

1 Palliativmedizin, 2. Medizinische Klinik, Universitäres Cancer Center Hamburg, Universitätsklinikum HamburgEppendorf

2 Palliativmedizinische Abteilung, Comprehensive Cancer Center CCC Erlangen-EMN, Universitätsklinikum Erlangen, Friedrich-Alexander-Universität Erlangen-Nürnberg

\section{Schlüsselwörter}

Angehörige, Unterstützung, Comprehensive Cancer Center, Palliativmedizin

Key words

family caregiver, support, counselling, Comprehensive Cancer Center, palliative care

online publiziert 20.08 .2021

\section{Bibliografie}

Dtsch Med Wochenschr 2021; 146: e74-e80

DOI 10.1055/a-1543-2511

ISSN 0012-0472

(C) 2021. The Author(s).

This is an open access article published by Thieme under the terms of the Creative Commons Attribution-NonDerivative-NonCommercial License, permitting copying and reproduction so long as the original work is given appropriate credit. Contents may not be used for commecial purposes, or adapted, remixed, transformed or built upon. (https://creativecommons.org/licenses/by-nc-nd/4.0/)

Georg Thieme Verlag KG, Rüdigerstraße 14,

70469 Stuttgart, Germany

Korrespondenzadresse

Prof. Dr. med. Karin Oechsle

Palliativmedizin, 2. Medizinische Klinik

Universitätsklinikum Hamburg-Eppendorf, Martinistr. 52,

20246 Hamburg, Deutschland

kaoechsl@uke.de

丹

Zusätzliches Material finden Sie unter

https://doi.org/10.1055/a-1543-2511

\section{ZUSAMMENFASSUNG}

Einleitung Eine leitliniengerechte onkologische Versorgung umfasst nicht nur die frühzeitige palliativmedizinische Mitbetreuung unheilbar erkrankter Patienten, sondern auch die Mitbetreuung ihrer Angehörigen. Untersuchungen zur Umsetzung im klinischen Alltag in Deutschland fehlen.

Methoden Die AG Palliativmedizin im Netzwerk der von der Deutschen Krebshilfe geförderten Onkologischen Spitzenzentren (CCC) führte mit einem selbstgestalteten Fragebogen eine systematische Erfassung aller Angebote in den CCC/in der spezialisierten Palliativversorgung (SPV) im CCC/lokal außerhalb des CCC an allen Standorten durch.

Ergebnisse Gut etabliert in den $17 \mathrm{CCC} /$ in der SPV sind die psychoonkologische (100\%/94\%), sozialrechtliche (94\%/ $100 \%)$ und seelsorgerische Beratung der Angehörigen (je 94\%) sowie Angebote für Kinder erkrankter Eltern ( $88 \%$ ) $100 \%$ ) und Informationsmaterialien (je $88 \%$ ). Häufiger als im restlichen CCC werden in der SPV pflegerische Schulungen (77\%/94\%) und Familienkonferenzen (59\%/88\%) durchgeführt. SOPs sind mit $23 \% / 18 \%$ selten etabliert, ebenso wie Screenings der Angehörigenbedürfnisse (0/24\%). Trauerund Selbsthilfegruppen sind mit je $82 \%$ häufiger außerhalb der CCC verfügbar. Die psychoonkologische/sozialrechtliche Beratung und Angebote für Kinder erkrankter Eltern (je 94\%) wurden als am wichtigsten für ein CCC eingeschätzt. Für die SPV waren es zudem die pflegerische Schulung sowie Informationsmaterialien (je 94\%). SOPs wurden mit $47 \% / 41 \%$ als sehr/extrem wichtig betrachtet, und Screenings der Angehörigenbedürfnisse mit $53 \% / 65 \%$.

Schlussfolgerung Psychosoziale/seelsorgerische Beratung der Angehörigen sowie Angebote für Kinder erkrankter Eltern sind in den CCC entsprechend ihrer Bedeutung gut etabliert, in der SPV auch pflegerische Schulungen und Familienkonferenzen. SOPs zur Mitbetreuung und Einbeziehung von Angehörigen sowie Screenings der Angehörigenbedürfnisse müssen dringend implementiert werden. 


\section{ABSTRACT}

Background According to current oncological guidelines, early integration of specialist palliative care (SPC) represents standard cancer care supporting not only the patients, but also their family caregivers. Data on the actual implementation in daily oncology practice in Germany are lacking.

Methods The Palliative Care Working Group of the network of Comprehensive Cancer Centers certified by the German Cancer Aid (CCC) assessed the implementation of measures for family caregiver support and involvement within the CCC/ within SPC in the CCC/local outside the CCC in all 17 CCC locations.

Results In the CCC/in SPC psycho-oncological (100\%/94\%), social (94\%/100\%) and spiritual counselling of family caregivers (94\% each) as well as support for children with parental cancer ( $88 \% / 100 \%$ ) and information materials for family caregivers ( $88 \%$ each) are well established. Training on nursing skills (77\%/94\%) and family conferences (59\%/88\%) are established more frequently within SPC than in the rest of the CCC. SOPs are rather rare (23\%/18\%) as well as screenings for family caregiver needs (0/24\%). Bereavement or self-help groups are with $82 \%$ each more frequent locally outside the CCC. Psycho-oncological and social counselling as well as support for children with parental cancer were scored as most important ( $94 \%$ each). For SPC, training on nursing skills and information materials were rated equally (94\% each). SOPs were rated as very/extremely important in $47 \% / 41 \%$ and routine screening for family caregiver in $53 \% / 65 \%$.

Conclusion In correspondence to their importance, psychosocial and spiritual counselling and support for children with parental cancer are well implemented in CCC. In SPC, training on nursing skills and family conferences are also well implemented. SOPs for family caregiver support and involvement as well as routine screenings for family caregiver needs have to be implemented urgently in the CCC.

\section{Einleitung}

Palliativmedizin richtet sich per definitionem nicht nur an Patienten mit einer lebensbedrohlichen Erkrankung, sondern nimmt auch ihre Angehörigen mit in den Fokus der Betreuung [1]. Dahinter steht das Verständnis, dass eine schwerwiegende Erkrankung nicht nur den Erkrankten selbst, sondern auch die Angehörigen, entweder verwandte oder andere nahestehende Personen, mit betrifft. Zahlreiche klinische Studien haben die Vielzahl an Problemen und unerfüllten Bedürfnissen von Angehörigen onkologischer Patienten, ihre hohe psychische Belastung und Morbidität sowie die zahlreichen ethischen Herausforderungen, vor denen sie im Erkrankungsverlauf der Patienten stehen, aufgezeigt [2-12]. Als besonders sensible Angehörigen-Subgruppe werden auch wiederholt die minderjährigen Kinder von an Krebs erkrankten Menschen und ihr spezifischer Versorgungsbedarf herausgestellt $[13,14]$. Erste Studien zeigen, dass die Situation der Angehörigen und auch der Patienten durch auf die Bedürfnisse der Angehörigen angepasste Mitbetreuungsangebote oder die Einbeziehung der Angehörigen in die Entscheidungs- und Versorgungsplanung verbessert werden kann [15-20]. Publikationen weisen zudem auf die deutliche Diskrepanz zwischen Leitlinienempfehlungen und klinischer Praxis sowie den nötigen Handlungsbedarf in Klinik und Forschung zur Verbesserung dieser Situation hin [21-25].

Studien haben außerdem gezeigt, dass eine frühzeitige Integration von Palliativmedizin in die Versorgung onkologischer Patienten nicht nur patientenbezogene, sondern auch Angehörigen-bezogene Parameter verbessern kann [26, 27]. Nationale und internationale Empfehlungen onkologischer Fachgesellschaften sowie onkologische Leitlinien sehen daher heute nicht nur die frühzeitige palliativmedizinische Mitbetreuung der Patienten ab Erstdiagnose einer unheilbaren Erkrankung, sondern auch der Angehörigen als Standard in einer modernen onkologischen Versorgung an [28-31]. Auch die nationale S3-Leitlinie „Palliativ- medizin bei Patienten mit einer nicht heilbaren Krebserkrankung“ sieht eine Mitbetreuung der Angehörigen frühzeitig nach Erstdiagnose einer unheilbaren Krebserkrankung vor. Dabei sollen „Angehörige über Therapieentscheidungen sowie die Behandlungs- und Versorgungsplanung informiert, in Gespräche zu Diagnose und Prognose einbezogen und ihnen Gelegenheit zur Mitgestaltung gegeben werden. Sie sollen außerdem entsprechend ihren Bedürfnissen und unter Berücksichtigung spezifischer und individueller Belastungsfaktoren im Erleben und in der Auseinandersetzung mit der Erkrankung des Patienten wahrgenommen, unterstützt und begleitet sowie über bestehende Unterstützungsangebote wie Selbsthilfegruppen und Angehörigenschulung informiert werden" [31].

Dennoch wurde bisher noch nicht systematisch untersucht, inwieweit diese Empfehlungen zur Mitbetreuung und Einbeziehung der Angehörigen im onkologischen Versorgungsalltag in Deutschland auch tatsächlich umgesetzt werden und entsprechende Angebote vorgehalten werden. Die von der Deutschen Krebshilfe e. V. geförderten Onkologischen Spitzenzentren (CCC) gelten als Paradebeispiele für eine besonders umfassende und qualitativ hochwertige onkologische Versorgung und sollten daher auch die Angehörigenmitbetreuung und -einbeziehung bestmöglich umgesetzt haben. Die Arbeitsgruppe (AG) Palliativmedizin im CCC-Netzwerk hat im Rahmen zusätzlicher Förderungen der Deutschen Krebshilfe e. V. seit ihrem ersten Netzwerktreffen 2011 schrittweise die Integration der Palliativmedizin in die CCC untersucht [32], Standard Operation Procedures (SOPs) zur integrierten Palliativversorgung [33] und Best-Practice-Empfehlungen entwickelt [34] sowie deren Implementierung überwacht [35, 36]. Im Rahmen eines zusätzlichen, 2020 von der Deutschen Krebshilfe e. V. bewilligten Arbeitspaketes für die AG Palliativmedizin im CCC-Netzwerk (Nr. 70113 949) soll nun unter anderem auch die Mitbetreuung und Einbeziehung der Angehörigen konkreter in die verschiedenen Förderziele mit aufgenommen werden. Um die aktuelle Situation der Umsetzung zu untersuchen 
und anschließend konkrete Maßnahmen zur Verbesserung der Angehörigenmitbetreuung in den CCC zu planen, wurden daher zunächst alle CCC-Standorte hinsichtlich ihrer aktuellen Mitbetreuung und Einbeziehung von Angehörigen in den Versorgungsalltag an den jeweiligen CCC sowie ihrer Einschätzung der Wichtigkeit verschiedener Angebote befragt.

\section{Methoden}

Ein Fragebogen wurde entworfen und unter allen Mitgliedern der AG Palliativmedizin zirkuliert, ergänzt und auf Vollständigkeit überprüft. Dieser selbst erstellte Fragebogen wurde noch nicht anderweitig publiziert oder eingesetzt. Er listet mögliche Angebote zur Unterstützung und Einbeziehung von Angehörigen im onkologischen Versorgungsalltag zur Auswahl auf: psychoonkologische Beratung/Betreuung der Angehörigen; sozialrechtliche Beratung; pflegerische Schulung/Beratung; ärztliche Sprechstunden für Angehörige; Familienkonferenzen; seelsorgerische Betreuung; Kunstund/oder Musiktherapie; Entspannungstechniken/Rückenschule/ Bewegungstherapie; Ernährungsberatung/Kochkurse für Angehörige; Angebote für Kinder erkrankter Eltern; Informationsmaterialien für Angehörige; SOPs zur Angehörigenbegleitung/-betreuung; Routine-Screening/-Assessment von Angehörigenbedürfnissen (wenn ja, mit welchem Instrument); Trauergruppen; Selbsthilfegruppen und andere (Freitext). Für jedes Item wurde gefragt, ob dieses Angebot im jeweiligen CCC-Standort vorliegt (ja/nein) und für wie wichtig das Mitglied der AG Palliativmedizin dieses Angebot auf einer 5-stufigen Skala von „nicht wichtig“ bis „extrem wichtig“ hält. Diese Abfrage wurde 3-fach durchgeführt - jeweils für die Angehörigen onkologischer Patienten im gesamten CCC, im Rahmen der spezialisierten Palliativversorgung (SPV) innerhalb des CCC sowie lokal außerhalb des CCC (hier wurden nur Aspekte erfragt, die aus dem CCC heraus beurteilbar waren). Zusätzlich wurden folgende Fragen mit aufgenommen, die jeweils mit ja/nein und „wenn ja, in welcher Form?“ beantwortet werden sollten: „Beziehen Sie Angehörige konkret in die (Weiter-) Entwicklung von Versorgungsstrukturen und/oder Unterstützungsangeboten mit ein?“, „Beziehen Sie Angehörige direkt in die Entwicklung von Forschungsprojekten mit ein?“, „Evaluieren Sie bereits Unterstützungsangebote für Angehörige, die an Ihrem CCC bestehen?" und "Kommen Angehörigen-bezogene Themen in der studentischen Lehre vor?" (kompletter Fragebogen siehe Anhang).

Im Mai 2020 wurden jeweils die Mitglieder der Arbeitsgruppe Palliativmedizin im CCC-Netzwerk aller 13 CCC bzw. bei mehreren Standorten eines CCC alle beteiligten Standorte, also insgesamt 17 CCC-Standorte, kontaktiert und um Teilnahme an der Befragung gebeten. Nach einer erneuten Erinnerung im Oktober 2020 lagen dann von allen 17 CCC-Standorten vollständig auswertbare Fragebögen vor. Diese wurden in eine SPSS-Datenbank eingegeben und deskriptiv ausgewertet.

\section{Ergebnisse}

Die von allen 17 CCC-Standorten ausgefüllten Fragebögen zeigten, dass in den CCC insgesamt bestimmte Angebote zur Mitbetreuung und Einbeziehung der Angehörigen flächendeckend gut etabliert sind, wie die psychoonkologische Beratung/Betreuung der Angehörigen (100\%), die sozialrechtliche und seelsorgerische Beratung (je $94 \%$ ) sowie Angebote für Kinder erkrankter Eltern und Informationsmaterialen für Angehörige (je $88 \%$ ). Andererseits bestehen nur in 4 CCC SOPs zu Angehörigenbegleitung und -betreuung (24\%) und in keinem CCC wird ein Routine-Screening von Angehörigenbedürfnissen durchgeführt.

Entsprechend dem multiprofessionellen Versorgungsansatz im Rahmen der SPV auf Palliativstationen oder auf Akutstation bei Mitbetreuung durch einen multiprofessionellen Palliativdienst finden sich dort innerhalb der CCC ebenfalls eine breit etablierte sozialrechtliche Beratung der Angehörigen (100\%), Angebote für Kinder erkrankter Eltern (100\%), eine psychoonkologische und seelsorgerische Beratung/Betreuung (je 94\%) und Informationsmaterialen für Angehörige (88\%). Häufiger als im restlichen CCC werden in der SPV pflegerische Schulungen/Beratungen (94\%), Familienkonferenzen (88\%) und Musik- und Kunsttherapie für Angehörige (82\%) im Rahmen der Regelversorgung durchgeführt. SOPs zur Angehörigenmitbetreuung liegen im Rahmen der SPV mit $3(18 \%)$ sogar in einem Standort weniger vor als im Rahmen des gesamten CCC (24\%). Dafür bestehen in 4 Standorten (24\%) in die SPV-Regelversorgung integrierte Routine-Screenings hinsichtlich der Bedürfnisse der Angehörigen (2-mal mittels des IPOS = „Integrated Palliative care Outcome Scale“ [37], 1-mal mittels MIDOS = „Minimales Dokumentationssystem für Patienten in der Palliativmedizin“ [38] und 1-mal mit einem selbst entwickelten Instrument), während sie im restlichen CCC an keinem Standort vorliegen.

Auch außerhalb der CCC bestehen lokal zusätzliche Angebote für Angehörige. Am häufigsten sind dies sozialrechtliche Beratungen (83\%), Trauergruppen und Selbsthilfegruppen für Angehörige (je $82 \%)$, psychologische Beratung (77\%) und Angebote für Kinder erkrankter Eltern (77\%). Trauergruppen und Selbsthilfegruppen für Angehörige bestehen derzeit also sogar häufiger außerhalb (je $82 \%$ ) als innerhalb der CCC (41-65\%). Zu Details zum Vorliegen von Angeboten zur Angehörigenmitbetreuung und -einbindung siehe $>$ Tab. 1 .

Von den jeweiligen Mitgliedern der AG Palliativmedizin in den verschiedenen CCC-Standorten wurden für die CCC insgesamt die psychoonkologische Beratung/Betreuung der Angehörigen, die sozialrechtliche Beratung sowie Angebote für Kinder erkrankter Eltern mit jeweils $94 \%$ am häufigsten als „sehr oder extrem wichtig“ eingeschätzt. Für die SPV waren es die psychoonkologische Beratung/Betreuung, die sozialrechtliche Beratung, die pflegerische Schulung/Beratung sowie Informationsmaterialien mit je $94 \%$. Dass solche Angebote auch außerhalb des CCC lokal bestehen, wurde mit $83 \%$ für die psychoonkologische Beratung, $77 \%$ für Angebote für Kinder erkrankter Eltern und 71 \% für die sozialrechtliche Beratung als sehr oder extrem wichtig erachtet. Die Wichtigkeit von Trauergruppen, die bisher häufiger außerhalb der CCC bestehen, wurde mit 65-71\% konstant innerhalb und außerhalb der CCC für wichtig erachtet. Für Selbsthilfegruppen für Angehörige galt dies mit 53-59\% ebenfalls. SOPs wurden mit $47 \%$ in den CCC insgesamt und mit $41 \%$ in der SPV relativ selten für sehr oder extrem wichtig erachtet, während dies für ein Routine-Screening der Angehörigenbedürfnisse mit $53 \%$ und $65 \%$ etwas höher war. Details zur Einschätzung der jeweiligen 
- Tab. 1 Vorliegen von Angeboten zur Mitbetreuung und Einbindung von Angehörigen ( $n=17)$.

\begin{tabular}{|c|c|c|c|}
\hline & $\begin{array}{l}\text { für Angehörige aller } \\
\text { onkologischen } \\
\text { Patienten im CCC }\end{array}$ & $\begin{array}{l}\text { für Angehörige } \\
\text { von Patienten } \\
\text { in der SPV im } \\
\text { CCC }\end{array}$ & $\begin{array}{l}\text { für Angehörige } \\
\text { lokal außerhalb } \\
\text { des CCC }\end{array}$ \\
\hline & $\%(n)$ & $\%(n)$ & $\%(n)$ \\
\hline psychoonkologische Beratung/Betreuung der Angehörigen & $100 \%(17)$ & $94 \%(16)$ & $77 \%(13)$ \\
\hline sozialrechtliche Beratung für Angehörige & $94 \%(16]$ & $100 \%(17)$ & $83 \%(14)$ \\
\hline pflegerische Schulung/Beratungen & $77 \%(13)$ & $94 \%(16)$ & $71 \%(12)$ \\
\hline ärztliche Sprechstunden nur für Angehörige & $35 \%(6)$ & $65 \%(11)$ & $18 \%(3)$ \\
\hline Familienkonferenzen & $59 \%(10)$ & $88 \%(15)$ & $24 \%(4)$ \\
\hline seelsorgerische Betreuung & $94 \%(16)$ & $94 \%(16)$ & $82 \%(14)$ \\
\hline Kunst- und/oder Musiktherapie & $47 \%(8)$ & $82 \%(14)$ & $24 \%(4)$ \\
\hline Entspannungstechniken/Rückenschule/Bewegungstherapie & $41 \%(7)$ & $53 \%(9)$ & $53 \%(9)$ \\
\hline Ernährungsberatung/Kochkurse für Angehörige & $59 \%(10)$ & $53 \%(9)$ & $35 \%(6)$ \\
\hline Angebote für Kinder erkrankter Eltern & $88 \%(15)$ & $100 \%(17)$ & $77 \%(13)$ \\
\hline Informationsmaterialien für Angehörige & $88 \%(15)$ & $88 \%(15)$ & n.e. \\
\hline SOP zur Angehörigenbegleitung/-betreuung & $24 \%(4)$ & $18 \%(3)$ & n.e. \\
\hline Routine-Screening/-Assessment von Angehörigenbedürfnissen & $0(0)$ & $24 \%(4)$ & n.e. \\
\hline Trauergruppen & $41 \%(7)$ & $59 \%(10)$ & $82 \%(14)$ \\
\hline Selbsthilfegruppen & $65 \%(11)$ & $59 \%(10)$ & $82 \%(14)$ \\
\hline Andere & 0 & 0 & 0 \\
\hline
\end{tabular}

Wichtigkeit von Angeboten zur Angehörigenmitbetreuung und -einbindung siehe $>$ Tab. 2 .

Ein Einbezug von Angehörigen in die (Weiter-) Entwicklung von Versorgungsstrukturen und/oder Unterstützungsangeboten besteht an 11 Standorten (65\%), in die Entwicklung von Forschungsprojekten an 7 Standorten (41\%) und in die Evaluation von Unterstützungsangeboten für Angehörige an 3 Standorten (19\%). Alle 17 Standorte (100\%) geben an, dass Angehörigen-bezogene Themen in ihrer studentischen Lehre vorkommen.

\section{Diskussion}

Die Befragung aller 17 Standorte hinsichtlich der Mitbetreuung und Einbeziehung von Angehörigen in die Regelversorgung onkologischer Patienten in den CCC zeigt, dass manche Angebote, wie psychoonkologische Betreuung und Beratung und sozialrechtliche und seelsorgerische Beratung sowie Angebote für Kinder erkrankter Eltern, in den CCC insgesamt und in der SPV der CCC gut etabliert sind. Die Angebote für Kinder erkrankter Eltern werden den Forderungen nach spezifischen Betreuungsangeboten für diese sensible Angehörigen-Subgruppe gerecht [13, 14]. Psychoonkologische und sozialrechtliche Beratung sowie Angebote für Kinder erkrankter Eltern wurden von Vertretern der Palliativver- sorgung an den CCC als am wichtigsten für ein CCC insgesamt eingeschätzt.

Diese Angebote versuchen der in Studien beschriebenen großen Zahl an Problemen, Belastungen, unerfüllten Bedürfnissen und hohen psychischen Morbidität der Angehörigen onkologischer Patienten gerecht zu werden [2-12]. Viele der Studien zeigen eine Vielzahl an Problemen, obwohl sie in CCC mit den oben genannten Angeboten durchgeführt wurden [4-7, 9, 10]. In einer eigenen Arbeit konnten wir zeigen, dass bei Erstaufnahme von onkologischen Patienten auf die Palliativstation eines CCC nur etwa $25 \%$ der Angehörigen zuvor - zumeist psychoonkologische - Unterstützung in Anspruch genommen hatten [10]. Es ist dementsprechend davon auszugehen, dass diese Angebote zwar in nahezu allen CCC-Standorten bestehen, wie es auch die S3-Leitinie „Palliativmedizin für Patienten mit einer nicht heilbaren Krebserkrankung" (Kapitel 5.5.7) vorgibt. Offen bleibt, wie gut den Angehörigen die Angebote bekannt sind, wie stark und von welchen Angehörigen die Angebote genutzt werden und welche Faktoren dazu führen, dass die Angebote nicht wahrgenommen werden. Solche Kenntnisse könnten helfen, die Angebote zielgruppenorientierter zu offerieren.

Trauer- und Selbsthilfegruppen wurden für innerhalb und außerhalb der CCC als gleich wichtig erachtet, werden aber häufiger durch externe Anbieter außerhalb der CCC angeboten. Dies 
- Tab. 2 Wichtigkeit der Angebote zur Mitbetreuung und Einbindung von Angehörigen ( $n=17)$.

\begin{tabular}{|c|c|c|c|}
\hline & $\begin{array}{l}\text { für Angehörige aller } \\
\text { onkologischen } \\
\text { Patienten im CCC }\end{array}$ & $\begin{array}{l}\text { für Angehörige } \\
\text { von Patienten } \\
\text { in der SPV im } \\
\text { CCC }\end{array}$ & $\begin{array}{l}\text { für Angehörige } \\
\text { lokal außerhalb } \\
\text { des CCC }\end{array}$ \\
\hline & $\begin{array}{l}\text { sehr/extrem wichtig } \\
\%(n)\end{array}$ & $\begin{array}{l}\text { sehr/extrem } \\
\text { wichtig \% (n) }\end{array}$ & $\begin{array}{l}\text { sehr/extrem } \\
\text { wichtig \% (n) }\end{array}$ \\
\hline psychoonkologische Beratung/Betreuung der Angehörigen & $94 \%(16)$ & $94 \%(16)$ & $83 \%(14)$ \\
\hline sozialrechtliche Beratung für Angehörige & $94 \%(16)$ & $94 \%(16)$ & $71 \%(12)$ \\
\hline pflegerische Schulung/Beratungen & $77 \%(13)$ & $94 \%(16)$ & $65 \%(11)$ \\
\hline ärztliche Sprechstunden nur für Angehörige & $47 \%(8)$ & $65 \%(11)$ & $41 \%(7)$ \\
\hline Familienkonferenzen & $77 \%(13)$ & $88 \%(15)$ & $53 \%(9)$ \\
\hline seelsorgerische Betreuung & $83 \%(14)$ & $77 \%(13)$ & $47 \%(8)$ \\
\hline Kunst- und/oder Musiktherapie & $41 \%(7)$ & $47 \%(8)$ & $30 \%(5)$ \\
\hline Entspannungstechniken/Rückenschule/Bewegungstherapie & $35 \%(6)$ & $47 \%(8)$ & $35 \%(6)$ \\
\hline Ernährungsberatung/Kochkurse für Angehörige & $23 \%(4)$ & $30 \%(5)$ & $24 \%(4)$ \\
\hline Angebote für Kinder erkrankter Eltern & $94 \%(16)$ & $88 \%(15)$ & $77 \%(13)$ \\
\hline Informationsmaterialien für Angehörige & $88 \%(15)$ & $94 \%(16)$ & n.e. \\
\hline SOP zur Angehörigenbegleitung/-betreuung & $47 \%(8)$ & $41 \%(7)$ & n.e. \\
\hline Routine-Screening/-Assessment von Angehörigenbedürfnissen & $53 \%(9)$ & $65 \%(11)$ & n.e. \\
\hline Trauergruppen & $65 \%(11)$ & $71 \%(12)$ & $71 \%(11)$ \\
\hline Selbsthilfegruppen & $53 \%(9)$ & $41 \%(7)$ & $59 \%(10)$ \\
\hline Andere & 0 & 0 & 0 \\
\hline
\end{tabular}

birgt die Gefahr, dass keine explizite und individuelle Einladung zu Trauerangeboten an Angehörige im Verlauf der Mitbetreuung im CCC erfolgt und sie deshalb nur begrenzt in Anspruch genommen werden, wie auch in der Literatur berichtet [21].

Im Rahmen der multiprofessionellen SPV werden Familienkonferenzen häufiger als im restlichen CCC durchgeführt. Sie sind eine hocheffektive Möglichkeit, Angehörige explizit in Therapieund Versorgungsentscheidungen sowie Prognosegespräche einzubinden. Diese Einbeziehung ist für die psychische Gesundheit, das Wohlbefinden und die Zufriedenheit der Angehörigen, aber auch die Versorgungsqualität der Patienten von großer Bedeutung $[18,19,25]$. Eine Stärkung von Familienkonferenzen in den CCC allgemein könnte die Einbeziehung Angehöriger demnach noch verbessern. Dies gilt ebenfalls für die Bereitstellung von pflegerischen Schulungen sowie Informationsmaterialien für Angehörige, die in der SPV häufiger durchgeführt und als sehr wichtig eingeschätzt werden. Fehlende erkrankungsbezogene Informationen sowie Informationen zum praktischen Umgang mit Symptomen und dem Verlauf der Erkrankung werden von vielen Angehörigen in zahlreichen Studien wiederholt als die am häufigsten unerfüllt gebliebenen Bedürfnisse angegeben [8, 10]. Verschiedene Studien haben die Effektivität von Angehörigenschulungen, inklusive pflegerischer Aspekte, psychologischer Belastungen und erkrankungsbezogener Informationen, sowohl als Präsenzschulungen [16, 26] als auch in Online-Formaten [15, 20, 22] für Angehörige aufzeigen können. Im deutschsprachigen Raum besteht aber derzeit noch ein Mangel an validierten und prospektiv evaluierten Angeboten.

Zahlreiche Studien weisen auf die große Bedeutung von Screening-Erhebungen der Angehörigenbedürfnisse im Versorgungsalltag hin [3, 8, 22], und auch die S3-Leitlinie „Palliativmedizin für Patienten mit einer nicht heilbaren Krebserkrankung“ (Kapitel 5.5.7) empfiehlt, dass „Angehörige entsprechend ihren Bedürfnissen und unter Berücksichtigung spezifischer und individueller Belastungsfaktoren [...] wahrgenommen [...] werden sollen. “ Dennoch zeigen unsere Ergebnisse, dass Routine-Screenings bezüglich der Angehörigenbedürfnisse in den CCC insgesamt gar nicht und in der SPV nur selten etabliert sind. Andererseits werden sie aber von $53 \%$ (CCC insgesamt) und $65 \%$ (SPV der CCC) der Befragten als jeweils sehr oder extrem wichtig betrachtet. Da bisher kein Standardinstrument zum Screening der Angehörigenbedürfnisse im deutschsprachigen Raum etabliert ist, wird es Aufgabe der AG Palliativmedizin im CCC-Netzwerk sein, dieses zu etablieren, zu validieren und in den CCC zu implementieren.

Bemühungen hin zur stärkeren Einbeziehung und bedürfnisorientierten Mitbetreuung von Angehörigen in den CCC zeigen die 
Ergebnisse der Erhebung beispielweise in der Einbeziehung Angehörigen-bezogener Themen in die Lehre, wie es auch den Europäischen Empfehlungen für Kernkompetenzen in der Palliativmedizin entspricht [40] und in einem hohen Anteil der Standorte, die Angehörige in die Weiterentwicklung von Versorgungsstrukturen und/oder Unterstützungsangeboten und in Forschungsprojekte im Sinne des „Participatory Action Research“ einbeziehen, praktiziert wird.

Neben einer verstärkten Einbindung von Angehörigen in die Evaluation von Unterstützungsangeboten könnte eine strukturelle Verankerung der Angehörigenmitbetreuung in SOPs erfolgen, wie sie auch zu verschiedenen Aspekten der Patientenversorgung in den CCC, sowohl in der SPV als auch in den CCC insgesamt, bereits etabliert [36] ist.

\section{Limitationen}

Die vorliegende Analyse zeichnet sich dadurch aus, dass sie ein flächendeckendes Bild der Angehörigenmitbetreuung und -einbeziehung in allen 17 Standorten der von der Deutschen Krebshilfe geförderten Onkologischen Spitzenzentren gibt. Diese werden aufgrund ihrer hohen multidisziplinären und multiprofessionellen Qualität in der Versorgung onkologischer Patienten ausgezeichnet. Es ist daher davon auszugehen, dass die Ergebnisse dieser Analyse nicht auf die flächendeckende Versorgung onkologischer Patienten in Deutschland übertragbar sind.

\section{Ausblick}

Die vorliegende Untersuchung zeigt, dass in den CCC bestimmte Angebote zur Unterstützung und Einbeziehung von Angehörigen gut etabliert sind. Dennoch zeigen Spitzenzentren noch Defizite in der Mitbetreuung und Einbeziehung von Angehörigen auf. Weitere Verbesserungen in den CCC im Rahmen der Förderung der Deutschen Krebshilfe könnten im Sinne des sogenannten „Outreachs“ in der flächendeckenden, breiten Versorgung onkologischer Patienten und ihrer Angehörigen zu Verbesserungen beitragen. Angehörigenschulungsangebote könnten im Rahmen der CCC, unter Rückgriff auf Angebote, die in SPV vorliegen, entwickelt und evaluiert und dann auch außerhalb der CCC zur Verfügung gestellt werden. Informationsmaterialien bestehen zwar bereits an den meisten Standorten, dennoch sollten umfassende, spezifische Broschüren, Schulungen und Online-Informations- und Trainingsangebote für Angehörige onkologischer Patienten weiterentwickelt und evaluiert werden. Ebenso könnte es Aufgabe der AG Palliativmedizin im CCC-Netzwerk sein, ein Standardinstrument zum Screening der Angehörigenbedürfnisse zu etablieren und in und außerhalb der CCC zu implementieren. Ein weiteres Ziel der AG ist es außerdem, zu den einzelnen Formen der Angehörigenmitbetreuung in den CCC und im Rahmen des „Outreachs“ auch flächendeckend nutzbare SOPs und Best-Practice-Empfehlungen zu formulieren.

Weiterhin untersucht werden sollten die Nutzung der Angebote und hinderliche Faktoren, ggf. mit besonderem Fokus auf die Nutzung und Verbesserung der Nutzung externer Trauerangebote. Diese Erkenntnisse können ebenfalls auch außerhalb der CCC zur Verbesserung der Unterstützung und Einbeziehung von Angehörigen beitragen.

\section{KERNAUSSAGEN}

- Psychosoziale und seelsorgerische Beratungen der Angehörigen sowie Angebote für Kinder erkrankter Eltern sind in den CCC insgesamt entsprechend ihrer Bedeutung gut etabliert.

- Im Rahmen der spezialisierten Palliativversorgung an den CCC sind es zusätzlich auch pflegerische Schulungen und Familienkonferenzen.

- Trauergruppen und Selbsthilfegruppen sollten häufiger innerhalb als bisher häufiger außerhalb der CCC angeboten werden.

- SOPs zur Mitbetreuung und Einbeziehung von Angehörigen sowie Routine-Screenings der Angehörigenbedürfnisse müssen in den CCC dringend implementiert werden.

\section{Interessenkonflikte}

Karin Oechsle hat Forschungsgelder der Deutschen Krebshilfe e. V. für das vorliegende Projekt (Bearbeitungsnummer 70113 949) erhalten sowie Forschungsgelder der Hamburger Krebsgesellschaft e. V. im Rahmen ihrer Stiftungsprofessur für Palliativmedizin mit Schwerpunkt Angehörigenforschung. Weitere Interessenkonflikte bestehen nicht. Tabea Theißen: keine Interessenkonflikte Maria Heckel: keine Interessenkonflikte Lisa Schwenzitzki: keine Interessenkonflikte Anneke Ullrich: keine Interessenkonflikte Christoph Ostgathe hat Forschungsgelder der Deutschen Krebshilfe e. V. für das vorliegende Projekt (Bearbeitungsnummer 70113 949) erhalten. Weitere Interessenkonflikte bestehen nicht.

\section{Danksagung}

Wir danken der Deutschen Krebshilfe e. V. für die Förderung des vorliegenden Projektes (Bearbeitungsnummer 70113949). Wir danken allen Mitgliedern der Arbeitsgruppe Palliativmedizin im Netzwerk der von der Deutschen Krebshilfe geförderten Onkologischen Spitzenzentren für die Teilnahme an dieser Befragung.

\section{Literatur}

[1] WHO. National cancer control programmes: policies and managerial guidelines. World Health Organization. 2002

[2] Dionne-Odom JN, Demark-Wahnefried W, Taylor RA et al. The self-care practices of family caregivers of persons with poor prognosis cancer: differences by varying levels of caregiver well-being and preparedness. Support Care Cancer 2017; 25: 2437-2444

[3] Laryionava K, Pfeil TA, Dietrich M et al. The second patient? Family members of cancer patients and their role in end-of-life decision making. BMC Palliat Care 2018; 17: 29

[4] Oechsle K, Ullrich A, Marx G et al. Psychological burden in family caregivers of patients with advanced cancer at initiation of specialist inpatient palliative care. BMC Palliat Care 2019; 18: 102

[5] Oechsle K, Ullrich A, Marx G et al. Prevalence and Predictors of Distress, Anxiety, Depression, and Quality of Life in Bereaved Family Caregivers of Patients With Advanced Cancer. Am J Hosp Palliat Care 2020; 37: 201213

[6] Preisler M, Heuse S, Riemer M et al. Early integration of palliative cancer care: patients' and caregivers' challenges, treatment preferences, and 
knowledge of illness and treatment throughout the cancer trajectory. Support Care Cancer 2018; 26: 921-931

[7] Rosenberger C, Höcker A, Cartus M et al. [Outpatient psycho-oncological care for family members and patients: access, psychological distress and supportive care needs]. Psychother Psychosom Med Psychol 2012; 62: $185-194$

[8] Sklenarova H, Krümpelmann A, Haun MW et al. When do we need to care about the caregiver? Supportive care needs, anxiety, and depression among informal caregivers of patients with cancer and cancer survivors. Cancer 2015; 121: 1513-1519

[9] Ullrich A, Ascherfeld L, Marx G et al. Quality of life, psychological burden, needs, and satisfaction during specialized inpatient palliative care in family caregivers of advanced cancer patients. BMC Palliat Care 2017; 16: 31

[10] Ullrich A, Marx G, Bergelt C et al. Supportive care needs and service use during palliative care in family caregivers of patients with advanced cancer: a prospective longitudinal study. Support Care Cancer 2021; 29 : $1303-1315$

[11] Ullrich A, Theochari M, Bergelt C et al. Ethical challenges in family caregivers of patients with advanced cancer - a qualitative study. BMC Palliat Care 2020; 19: 70

[12] Weißflog G, Hönig K, Gündel H et al. Associations between dyadic coping and supportive care needs: findings from a study with hematologic cancer patients and their partners. Support Care Cancer 2017; 25: $1445-1454$

[13] Morris JN, Martini A, Preen D. The well-being of children impacted by a parent with cancer: an integrative review. Support Care Cancer 2016; 24: 3235-3251

[14] Shah BK, Armaly J, Swieter E. Impact of Parental Cancer on Children. Anticancer Res 2017; 37: 4025-4028

[15] Benson J], Oliver DP, Washington KT et al. Online social support groups for informal caregivers of hospice patients with cancer. Eur J Oncol Nurs 2020; 44: 101698

[16] Hendrix CC, Bailey DE Jr, Steinhauser KE et al. Effects of enhanced caregiver training program on cancer caregiver's self-efficacy, preparedness, and psychological well-being. Support Care Cancer 2016; 24: 327-336

[17] Kent EE, Mollica MA, Dionne-Odom JN et al. Effect of instrumental support on distress among family caregivers: Findings from a nationally representative study. Palliat Support Care 2020; 18: 519-527

[18] Laidsaar-Powell R, Butow P, Bu S et al. Family involvement in cancer treatment decision-making: A qualitative study of patient, family, and clinician attitudes and experiences. Patient Educ Couns 2016; 99: 11461155

[19] Okado I, Pagano I, Cassel K et al. Perceptions of care coordination in cancer patient-family caregiver dyads. Support Care Cancer 2021; 29: 2645-2652

[20] Wittenberg E, Xu J, Goldsmith J et al. Caregiver Communication About Cancer: Development of a mhealth resource to support family caregiver communication burden. Psychooncology 2019; 28: 365-371

[21] Aoun SM, Rumbold B, Howting D et al. Bereavement support for family caregivers: The gap between guidelines and practice in palliative care. PloS one 2017; 12: e0184750-e0184750

[22] Kent EE, Rowland JH, Northouse L et al. Caring for caregivers and patients: Research and clinical priorities for informal cancer caregiving. Cancer 2016; 122: 1987-1995

[23] Lambert SD, Ould Brahim L, Morrison M et al. Priorities for caregiver research in cancer care: an international Delphi survey of caregivers, clinicians, managers, and researchers. Supportive Care Cancer 2019; 27 : 805-817
[24] Selman LE, Brighton L], Sinclair S et al. Patients' and caregivers' needs, experiences, preferences and research priorities in spiritual care: A focus group study across nine countries. Palliat Med 2018; 32: 216-230

[25] Thomas TH, Campbell GB, Lee Y] et al. Priorities to improve cancer caregiving: report of a caregiver stakeholder workshop. Support Care Cancer 2021; 29: 2423-2424

[26] Dionne-Odom JN, Azuero A, Lyons KD et al. Benefits of Early Versus Delayed Palliative Care to Informal Family Caregivers of Patients With Advanced Cancer: Outcomes From the ENABLE III Randomized Controlled Trial. J Clin Oncol 2015; 33: 1446-1452

[27] Hui D, Hannon BL, Zimmermann C et al. Improving patient and caregiver outcomes in oncology: Team-based, timely, and targeted palliative care. CA Cancer J Clin 2018; 68: 356-376

[28] Ferrell BR, Temel JS, Temin S et al. Integration of Palliative Care Into Standard Oncology Care: American Society of Clinical Oncology Clinical Practice Guideline Update. J Clin Oncol 2017; 35: 96-112

[29] Jordan K, Aapro M, Kaasa S et al. European Society for Medical Oncology (ESMO) position paper on supportive and palliative care. Ann Oncol 2018; 29: 36-43

[30] Kaasa S, Loge JH, Aapro M et al. Integration of oncology and palliative care: a Lancet Oncology Commission. Lancet Oncol 2018; 19: e588e653

[31] S3-Leitlinie Palliativmedizin für Patienten mit einer nicht heilbaren Krebserkrankung AWMF-Registernummer: 128/001OL 2015.

[32] Berendt ], Oechsle K, Thomas M et al. [State of integration of palliative care at Comprehensive Cancer Centers funded by German Cancer Aid]. Dtsch Med Wochenschr 2016; 141: e16-e23

[33] Stachura P, Berendt ], Stiel S et al. [Standard operating procedures (SOPs) for palliative care: Presence and relevance of palliative SOPs within the network of German Comprehensive Cancer Centers (CCCs) funded by the German Cancer Aid]. Schmerz 2017; 31: 47-53

[34] Berendt J, Stiel S, Simon ST et al. Integrating Palliative Care Into Comprehensive Cancer Centers: Consensus-Based Development of Best Practice Recommendations. The oncologist 2016; 21: 1241-1249

[35] Gahr S, Lödel S, Berendt J et al. Implementation of Best Practice Recommendations for Palliative Care in German Comprehensive Cancer Centers. The oncologist 2020; 25: e259-e265

[36] Lödel S, Ostgathe C, Heckel M et al. Standard Operating Procedures (SOPs) for Palliative Care in German Comprehensive Cancer Centers - an evaluation of the implementation status. BMC Palliat Care 2020; 19: 62

[37] Murtagh FE, Ramsenthaler C, Firth A et al. A brief, patient- and proxyreported outcome measure in advanced illness: Validity, reliability and responsiveness of the Integrated Palliative care Outcome Scale (IPOS). Palliat Med 2019; 33: 1045-1057

[38] Stiel S, Matthes ME, Bertram L et al. [Validation of the new version of the minimal documentation system (MIDOS) for patients in palliative care: the German version of the edmonton symptom assessment scale (ESAS)]. Schmerz 2010; 24: 596-604

[39] Yıldırım S, Kazaz SN, Semiz HS et al. An Evaluation of the Information Sources of Cancer Patients' Relatives. A Prospective Survey. J Cancer Educ 2019; 34: 913-919. doi:10.1007/s13187-018-1395-8

[40] Krumm N, Schmidlin E, Schulz C et al. Kernkompetenzen in der Palliativversorgung - ein Weißbuch der European Association for Palliative Care zur Lehre in der Palliativversorgung. Zeitschrift für Palliativmedizin 2015; 16: $152-167$ 